\title{
Research on the Algorithm Optimization of Improved Ant Colony Algorithm- LSACA
}

\author{
Yunheng Liu \\ School of information technology, Nanjing Forest Police College, Nanjing, \\ Jiangsu, China \\ lyhtzx@sohu.com
}

\begin{abstract}
The ant colony algorithm is an algorithm which is used to find the optimal path. As a kind of bionic evolutionary algorithm, the ant colony algorithm is inspired by the real ant colony foraging mechanisms. Firstly, this paper introduces the basic model of the ant colony algorithm. Then, aiming at the shortcomings of the ant colony algorithm, we propose a new probability formula of the optimal path and the new formula of the pheromone update. In addition, we combine the traditional ant colony algorithm with the local search algorithm and propose the improved ant colony algorithm. It is the LSACA algorithm. In the experimental analysis, we set and analyze the parameters of the algorithm. Then, we compare with the traditional algorithm to prove the feasibility and the effectiveness of the algorithm.
\end{abstract}

Keywords: The ant colony algorithm; Local search algorithm; Algorithm optimization

\section{Introduction}

The ant colony algorithm was proposed by the Italian scholar M.Dorigo and V.Maniezzo in 20 century [1-3]. The algorithm was a kind of heuristic evolutionary algorithm. It was proposed by simulating the optimal behavior of the ants in nature. According to studying the foraging behavior of the ants, they found that the ants collaborate by the chemicals which were called pheromones and formed the positive feedback. This made more and more ants gather to the shortest path. The emergence of the ant colony algorithm has aroused the great attention of the scholars. In the last twenty years, the ant colony algorithm has applied widely in the fields of combinatorial optimization, function optimization, system identification, network routing, robot path planning, data mining and integrated wiring of the large scale integrated circuit design etc. And it achieved the good results.

Walid Tfaili, Patrick Siarry introduced a new ant colony algorithm aimed at continuous and dynamic problems [4]. The algorithm was based on a continuous ant colony algorithm that uses a weighted continuous Gaussian distribution, instead of the discrete distribution, used to solve discrete problems. Jia Yu, Yun Chen, Jianping Wu presented an integrated modeling method for multi-criteria land-use suitability assessment(LSA) using classification rule discovery (CRD) by ant colony optimisation (ACO) in ArcGIS [5]. This new attempt applies artificial intelligent algorithms to intelligentise LSA by discovering suitability classification rules. R.F. Tavares Neto and M. Godinho Filho addressed the scheduling problem of minimizing the makespan in a permutational flowshop environment with the possibility of outsourcing certain jobs by means of the development of an ant colony optimization-based algorithm [6]. They called this new algorithm as flowshop ant colony optimization which is composed of two combined ACO heuristics. Bolun Chen, Ling Chen and Yixin Chen presented a feature selection algorithm based on ant colony optimization (ACO)[7]. They proposed a novel algorithm in which the artificial ants traversed on a directed graph with only $\mathrm{O}(2 \mathrm{n})$ arcs. The 
algorithm incorporated the classification performance and feature set size into the heuristic guidance, and selected a feature set with small size and high classification accuracy. Rana Forsati etc presented a new variant of ant colony optimization (ACO) which was called enriched Ant Colony Optimization (RACO) [8]. This variation tried to consider the previously traversed edges in the earlier executions to adjust the pheromone values appropriately and prevent premature convergence. In order to show the efficacy of the proposed algorithm, RACO was then applied to the feature selection problem. The results indicated that the proposed algorithms have superiorities over competitors. In addition, there were many foreign scholars conducting the research of the ant colony algorithm [9-14].

In China, there were many scholars studying the ant colony algorithm. Ni Qiangjian reviewed the research progress of the ant colony algorithm. Firstly, the author introduced the basic principle and the working mechanism of the ant colony algorithm. Then, the author reviewed the basic theory and the research status of the ant colony algorithm. It mainly included the parameters setting, the improvement and the application in combinatorial optimization problem and continuous optimization problem. On the basis, he gave the research focus and the developed erection. At last, it was the research outlook and the challenges of the ant colony algorithm [15]. According to the characteristics of the ant colony algorithm, Huang Han and other people proposed the convergence speed analysis theory of the ant colony algorithm which was based on the mathematical model of the absorption state. The author gave some theory method for estimating the expected convergence time of the ant colony algorithm. The author used the famous ACS algorithm to research the specific case. Based on the convergence speed analysis theory, the author proposed definition methods of the ACO-difficult and ACO-easy. Finally, the author used the ACS algorithm to solve the experimental data of the TSP problem. The author verified the conclusion and got the preliminary design guiding principle of the algorithm [16]. Xia Yamel proposed a kind of ant colony algorithm of the multi-pheromone updating. It was the MPDACO. It included the local optimal algorithm and the global optimal algorithm. The algorithm was based on the service composition model. The author studied and improved on the basis of the basic ant colony algorithm. The experimental results showed that the algorithm in this paper had better performance than the basic ant colony algorithm and the genetic algorithm which had a kind of service application [17]. Duan Haibin and other people proposed a kind of improved ant colony algorithm which was applied to solve the optimal problem in continuous space. They divided the solution vector of the continuous space optimal problem into finite grids. At the same time, they constructed an evaluation function which was related to the transition probability of the ant colony. And they improved the ant colony algorithm by the meeting search strategy in order to improve the global convergence performance for the improved ant colony algorithm [18]. Liu Changan improved the ant colony algorithm and proposed the convergence according to the target adaptive to adjust the heuristic function. Then, the author used the allocation principles of the wolves to update the pheromone and avoided to trap the local optimum. At the same time, the author proposed to use the particle swarm optimization algorithm to optimize and select the important parameters for the improved ant colony algorithm [19]. In addition, there were many domestic scholars studying the ant colony algorithm [20-23].

In this paper, we put forwards an improved ant colony algorithm. The algorithm is LSACA algorithm. The algorithm proposes a new optimal path probability formula and the new pheromone updating formula. And we combine the traditional ant colony algorithm with the local search algorithm. The algorithm improves the performance. The structure of this paper is as follows. The first part is the introduction. In this part, we introduce the status of the ant colony algorithm. The second part is the principle and the basic model of the ant colony algorithm. In this part, we introduce the basic principle and the model. The third part is the improved ant colony algorithm-LSACA. In the third part, 
we propose the improved ant colony algorithm and introduced the process of the algorithm. The fourth part is the experimental analysis. The last part is the conclusion.

\section{The Principle and the Basic Model of the Ant Colony Algorithm}

Ant colony algorithm is also called the ant algorithm. It is a probability algorithm which is used to find the optimal path in the graph. The inspiration of this algorithm is from the ants. During the process of the ants searching the food, they develop a path. The ant colony algorithm is a kind of simulated evolutionary algorithm.

The behavior of one ant is very simple. At the beginning, the path that the ants find may not be the shortest. But, with the passage of the time, more and more ants move cooperatively. And the path of the ants becomes shorter and shorter. Then, they get the shortest path. The researchers discover that when the ants move, they will leave a chemical substance. And the chemical substance is called the pheromone. These ants will release a certain of pheromone every time. The pheromone will evaporate with the time. When the ants choose the path, they will select a path randomly to go if the path is never traveled. At the same time, they release the pheromone which is related to the length of the path. The longer the path that the ants go is, the smaller the pheromone releases. The ants which are in the same group can perceive the existence and the strength of the pheromone. When the later ants meet the intersection, they will select their own direction according to the concentration of the pheromone. The more the pheromone is, the bigger the path chooses. Over and over, it forms a positive feedback mechanism. In general, in the same interval, the shorter the path is, the more the ants choose. Therefore, the pheromone which is in the shorter path is accumulated. The probability that the later ants choose is increased. After a period of time interval, the shortest path is selected. This is the reason that when there are many paths between the nest and the food, the ants can find the shortest path according to the collaboration. This is the principle of the ant colony algorithm.

The ant colony algorithm

Firstly, we define the parameters of the ant colony algorithm.

Table 1. The Parameters of the Ant Colony Algorithm

\begin{tabular}{|c|c|}
\hline The parameter & The meaning of the parameter \\
\hline$M$ & the number of the ants \\
\hline$m$ & The pheromone concentration of path $i \rightarrow j$ \\
\hline$\tau_{i j}$ & the maximum value of the pheromone concentration \\
\hline$\tau_{\max }$ & the minimum value of the pheromone concentration \\
\hline$\tau_{\min }$ & the probability of the path that the ants select from $i$ to $j$ \\
\hline$\theta_{i j}^{\alpha}$ & inspired value \\
\hline$\eta_{i j}^{\beta}$ & constant evaporation rate \\
\hline$\rho$ & iterations \\
\hline$N$ &
\end{tabular}




\begin{tabular}{|c|c|}
\hline$N_{\max }$ & the maximum iterations \\
\hline$\pi_{1 b}$ & the optimal value after one iteration \\
\hline$\pi_{g b}$ & the global value after the iterations \\
\hline$\Delta\left(\tau_{i j}\right)$ & the changed value of the pheromone concentration of $\tau_{i j}$ \\
\hline$d_{i j}$ & Euclidean distance between $i \rightarrow j$ \\
\hline$\eta_{i j}$ & the inspired function \\
\hline$P_{i j}$ & the state transition probability from $i$ to $j$ \\
\hline
\end{tabular}

The model of the any colony algorithm is described as follows.

We put randomly $M$ ants into one city. At the initial time, the amount of information in each path is equal.

$\tau_{i j}(t)=Q$

(1)

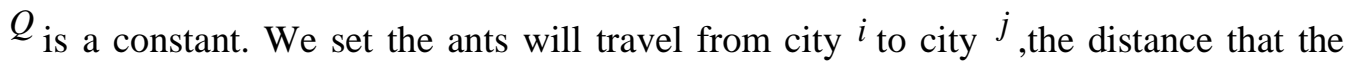
ants will travel is

$d_{i j}=\sqrt{\left(x_{i}-x_{j}\right)^{2}+\left(y_{i}-y_{j}\right)^{2}}$

(2)

So, the ants need to find a shortest path that they travel for all cities only once.

When the ants move, the amount of information in each path determines the transferred direction. We use the Tabu table $T B$ to represent the memory function of the ants. The Tabu table $T B$ records the cities that the ants have travelled. The set of cities changes with the change of the Tabu table. In the moving process of the ant colony, the intensity of the pheromone will change. Specifically, in the process of finding the path, the state transition probability of the ants $P_{i j}$ is calculated by the amount of the information and the inspired function. At $t$ time, the state transition probability $P_{i j}$ from the element city to the element city is calculated by the following function.

$P_{i j}(t)= \begin{cases}\frac{\left[\tau_{i j}\right]^{\alpha} \cdot\left[\eta_{i j}\right]^{\beta}}{\sum_{s \in(C-T B)}\left[\tau_{i j}\right]^{\alpha} \cdot\left[\eta_{i j}\right]^{\beta}}, \text { if } & j \in(C-T B) \\ 0 \quad, & \text { otherwise }\end{cases}$

$\alpha$ is the information inspiration factor. And $\beta$ is the expect inspiration factor.

$T B$ is the tabu table. It represents the set of the cities that the ants have visited. $C$ is the set of the cities which has the connections with other cities. $C-T B$ represents the city that the ants choose in the next step. According to the heuristic function, the smaller the two cities are, the bigger the heuristic function is. And the value of the state transition 
probability is bigger. If the pheromone in the path increases, the residual information will be too much. Then, the residual information will submerge the heuristic information. Therefore, after one ant select one city, or one ant traverses one city, namely one cycle ends. The residual information need to be undated. The amount of information can calculate as follows.

$\tau_{i j}(t+n)=(1-\rho) \tau_{i j}(t)+\Delta \tau_{i j}$

(4)

$$
\Delta \tau_{i j}=\sum_{m=1}^{M} \Delta \tau_{i j}
$$

$\rho_{\text {represents the residual coefficient of the pheromone. The influenced degree among }}$ the ants will be reflected. $(1-\rho)$ represents the evaporation degree of the pheromone between $t$ and $t+n . \Delta \tau_{i j}$ is the left amount of information in the path. And it is the incremental sum of the pheromone from the path $i_{\text {to }} j$.

\section{The Improved Ant Colony Algorithm-LSACA.}

The ant colony algorithm has the following advantages.

Firstly, the ant colony algorithm is a simulated evolutionary algorithm. It combines the positive feedback mechanism, the distributed computing with the greedy search algorithm. Therefore, during the search, the ant colony algorithm will not fall into the local optimum. At the same time, its best fitness function is not continuous and not planning. It can find out the optimal solution by the higher probability. In addition, the greedy search can find out the feasible solution quickly. It reduces the search time significantly.

Secondly, the ant colony algorithm is different from other algorithms. According to the natural evolution mechanism, it can show the complex phenomenon. This algorithm has the character of the higher scalability rely on the pheromone cooperation, not the information changed. In addition, it can deal with the difficult problem reliably and quickly.

Thirdly, the ant colony algorithm has a good parallel and computing. And it is very suitable for the huge amount of computation. When finding the optimal solution, the ant colony algorithm is easy to the standstill. After searching a certain period of time, the solution tends to be uniform. Therefore, it cannot search the optimal solution. And the easy solution is difficult to be found.

\subsection{The probability function of finding optimization}

In the improved ant colony algorithm, the ants choose the path according to the density of the pheromone and the heuristic information. For any $m$, the probability of choosing $j$ is as follows.

$P_{i j}= \begin{cases}\frac{\theta_{i j}^{\alpha} \eta_{i j}^{\beta}}{\sum_{j \in(C-T B)} \theta_{i j}^{\alpha} \eta_{i j}^{\beta}} & \text { if } \quad j \in(C-T B) \\ 0 & \text { otherwise }\end{cases}$

(6)

Where, $\alpha$ is the information inspiration factor. And $\beta$ is the expect inspiration factor. 
$\alpha$ represents the relative importance of the pheromone. It reflects that the influence of the pheromone on choosing the path in the process of moving. The bigger the value is, the bigger the pheromone is. It shows that the latter ants are influenced by the previous ants. And it can reflect the cooperation ability among the ants.

$\beta$ is the expect inspiration factor. ${ }^{\beta}$ represents the related importance of the visibility. It reflects the influence of the heuristic information on the path of the ants. The bigger the value is, the more closer to the greedy rule the state transition process is.

\subsection{The Updated Pheromone}

In order to prevent the excessive accumulation of the pheromone, the disappearance rate of the pheromone is a constant value. In order to improve the convergence speed, we use rotation method to enhance the pheromone of $\pi_{1 b}$ and $\pi_{g b}$.

$\tau_{i j}(t+1)=(1-\rho) \tau_{i j}(t)+\Delta\left(\tau_{i j}\right)$

(7)

$\rho_{\text {is a constant evaporation rate. }} \Delta\left(\tau_{i j}\right)$ is leaved pheromone in the path $(i, j)$ for the ants. ${ }^{\Delta\left(\tau_{i j}\right)}$ is reinforcementfor the global best or local best solution. We define as follows.

$\Delta\left(\tau_{i j}\right)=\frac{1}{f_{\text {best }}}$

Among them, $f_{\text {best }}$ is the target value for the local optimal solution or the global optimal solution.

When we reinforce the global optimal solution, the ant colony will concentrate on the solution. And it will lead to the poor convergence. Under the local optimal solution, different iterations have different values. Therefore, enhancing $\pi_{1 b}$ can make the pheromone increases in much more solutions. In the ant colony algorithm, the pheromone on the paths of $\pi_{1 b}$ increases after $N$ iterations. At this time, if the number of the iterations increases one time, the pheromone on the paths of $\pi_{g b}$ increases. In order to prevent the distance of the pheromone between the optimal solution and other solutions is

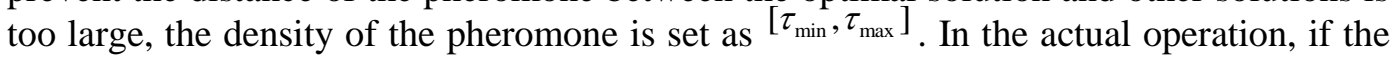
density of the pheromone is greater than ${ }^{\tau_{\max }}$, then density adjusts automatically as $\tau_{\max }$. Similarly, $\mathrm{f}$ the density of the pheromone is less than $\tau_{\max }$, then density adjusts automatically as $\tau_{\min }$.

We define ${ }^{\tau_{\min }}$ as follows.

$\tau_{\min }=\frac{1}{(1-\rho) f\left(\pi_{g b}\right)}$

(9)

$\rho_{\text {is a constant evaporation rate. }} f\left(\pi_{g b}\right)$ the target value of the global optimal solution.

We define ${ }^{\tau_{\min }}$ as follows.

$\tau_{\min }=\frac{\tau_{\max }(1-\sqrt[e]{c})}{\left(\frac{e}{2}-1\right) \sqrt[e]{c}}$

Among them, $e$ is the number of types. ${ }^{c}$ is a constant. If $e$ is small relatively, the problem is easy to deal with. If $e$ is big relatively, the problem is difficult to deal with. If 
$e$ is small relatively, $\tau_{\min }$ is big relatively. Then, ${ }^{\left[\tau_{\min }, \tau_{\max }\right]}$ is small relatively. If $e$ is big relatively, ${ }^{\tau_{\min }}$ is small relatively. Then, ${ }^{\left[\tau_{\min }, \tau_{\max }\right]}$ is big relatively.

\subsection{Local Search}

2-opt method can change the loop from the crossed road the convex road. The treatment process is as Figure 1. Using this method can optimize the solution. It does the preparatory work to find out the shortest path. Then, it updates globally the pheromone. The method will take a long time especially when the number of the ants is large. This method not only increases the time of the ant colony algorithm, but also influences the performance of the whole algorithm. This is the reason that the above algorithm needs a long time complexity. Therefore, solving the solution can save a lot of unnecessary waste of time.

According to the limitation of the above local search, we can use the following methods. Firstly, after each iteration of the ants, we order the paths that the ants search according to the sequence from the short to the long. Then, we cut out the half of front to do the local search.

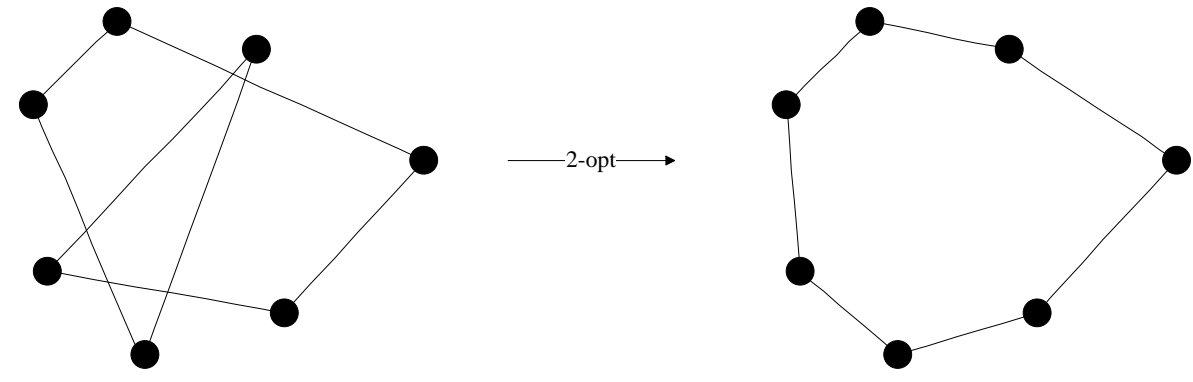

Figure 1. The 2-opt Processing Procedure

In each iteration of the ant colony algorithm, when each ant does the local search, not all the paths of the ants are useful for the optimal solution. Especially for those ants that have the long paths, the local search results are no use for the current solutions. In general, after each iteration, the optimal solution can be searched from the shorter paths according to the local search. If we use the 2-opt method to do the local search the paths for all of the ants, it must be consume a lot of time to search the no effect paths. Therefore, in the ant colony algorithm, after each iteration, the ants will do the local search for those short paths. It can not only reduce the waste of time, but also improve the efficiency of the search on the original basis.

The flow chart of LSACA is as the Figure 2 


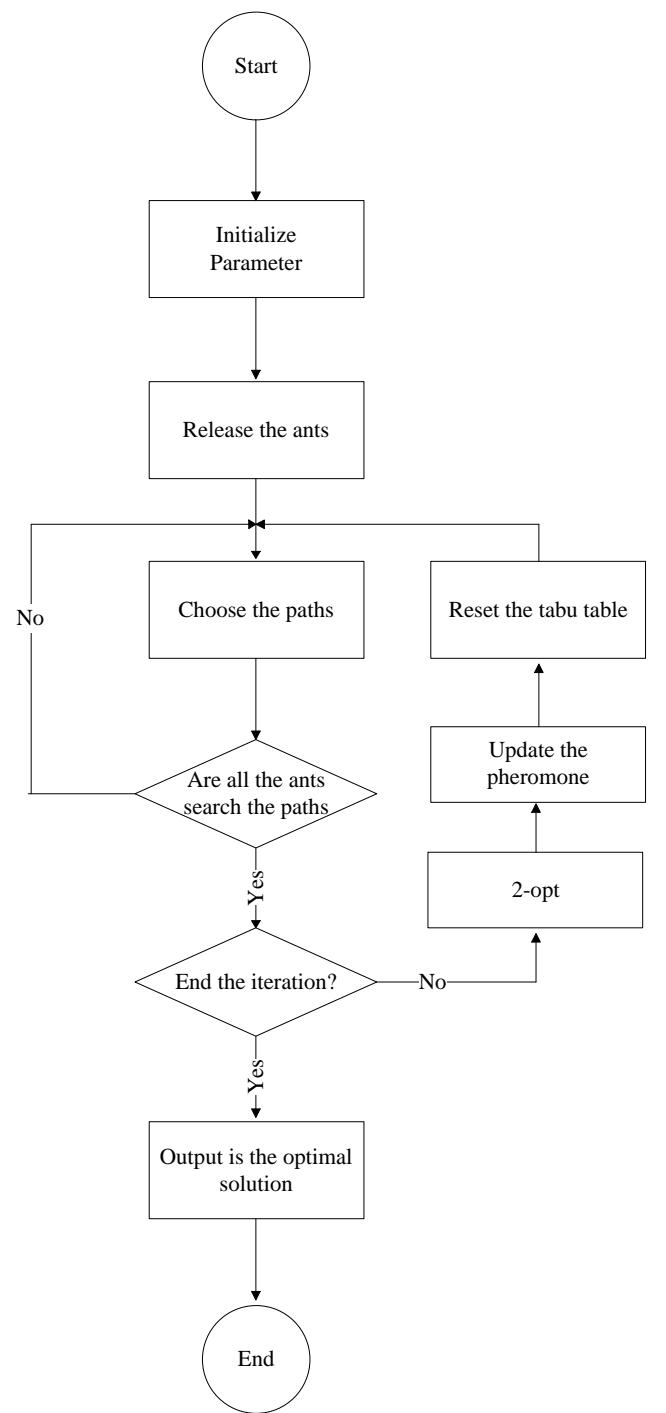

Figure 2. The Flow Chart of LSACA

The first step is to initialize the parameters. The number of the iteration is $N$. The density of the pheromone from the path $i \rightarrow j$ is $\tau_{i j}$. The inspired function is $\eta_{i j}$. The current path information table is $T a b u$. The optimal path length is $L_{-} b e s t$. The distance from $i_{\text {to }} j_{\text {is }} d_{i j}$.

The second step is to set the initial position of the ants. We initialize the Tabu table of the ants. We put all of the ants $M$ randomly.

The third step is to construct the solutions. According to the state change rules, each ant constructs a solution. Then, they generate a line. The ant visits all cities. And after one visit, the ant must be back to the origin. We assume the current point that the ant is located is $i$. However, the ants must follow the state change rule of function (6) to migrate continually from the point $i$ to the point $j$. They choose the next point according to different probability.

The fourth step is to check the completion of the construction solution. We check whether all of the ants complete the construction solutions. If they complete, we do the step 5. If not, we do the step 3. 
The fifth step is to construct the solution according to using the 2-opt method to optimize locally the ant algorithm. We compare all of the paths that the ants go. Then we get the path sequence from the short to the long. Then we select the half of the front to do the 2-opt local optimization. Finally, it shortens the length of the solution and quickens the convergence speed of the ant algorithm.

The sixth step is to update the pheromone. Then we compare the current shortest paths and adopt the function (9-10) to update the pheromone.

The seventh step is to terminate the algorithm. If the termination condition is satisfied, the algorithm ends. Otherwise, $N=N+1, N \leq N_{\max }$ and we transfers to the step 2 .

\section{Experiment}

In order to verity the performance of the algorithm, we use the actual cases to do the experiment. We studied the parameters of the LSACA algorithm. Firstly, we studied the range of the pheromone density. The range was $\left[\tau_{\min }, \tau_{\max }\right]$. The results were as follows. Among them, the "Best", "Avg" and "Worst" expressed the best solution, the average solution and the worst solution in the 30 operations.

Table 2. The Influence of $\left[\tau_{\min }, \tau_{\max }\right]$ on the Results of LSACA

\begin{tabular}{|c|c|c|c|}
\hline$\left[\tau_{\min }, \tau_{\max }\right]$ & Best & Worst & Avg \\
\hline 1,10 & 378 & 402 & 380.5 \\
\hline 1,20 & 378 & 399 & 376.4 \\
\hline 1,30 & 382 & 404 & 385.6 \\
\hline 1,40 & 386 & 407 & 389.7 \\
\hline 2,10 & 389 & 410 & 396.1 \\
\hline 2,20 & 395 & 413 & 398.2 \\
\hline 2,30 & 398 & 415 & 404.5 \\
\hline 2,40 & 402 & 418 & 408.4 \\
\hline
\end{tabular}

From the above table, we can see that when the range of pheromone density is in $[1,20]$, LSACA algorithm has the higher performance. Therefore, the range is set as $[1,20]$.

Then, we verify the performance of the LSACA algorithm. We combine the results of the LSACA algorithm with the ant colony algorithm. The parameter is $\left[\tau_{\min }, \tau_{\max }\right]=[1,20]$. $c=0.05, \alpha=1$ and $\beta=3$. The experimental results are as follows.

Table 3. The Comparison between of LSACA and ACA

\begin{tabular}{|c|c|c|c|c|c|c|}
\hline \multirow{2}{*}{ Instance } & \multicolumn{2}{|c|}{ Best } & \multicolumn{2}{c|}{ Avg } & \multicolumn{2}{c|}{ Worst } \\
\cline { 2 - 7 } & LSACA & ACA & LSACA & ACA & LSACA & ACA \\
\hline kroA100 & 21132 & 21230 & 21799.3 & 21980.4 & 21257 & 22736 \\
\hline kroE100 & 22032 & 22786 & 22478.4 & 23246.1 & 22935 & 23761 \\
\hline berlin52 & 7321.4 & 7576.3 & 7438.2 & 7653.9 & 7653.8 & 7732.9 \\
\hline ch150 & 6320.1 & 6421.8 & 6403.1 & 6482.5 & 6474.5 & 6536.2 \\
\hline d198 & 15332 & 15392 & 15377.5 & 15435.7 & 15420 & 15487 \\
\hline
\end{tabular}

From the above table, we can see that the performance of LSACA algorithm is better than the ant colony algorithm. According to the experiment, we can see that the LSACA algorithm is superior to the ACA algorithm. Therefore, we verify the effectiveness and the feasibility of the LSACA algorithm. 


\section{Conclusion}

Since the appearance of the ant colony algorithm, many scholars have improved the algorithm. In this paper, we studied the ant colony algorithm and proposed the improved the ant colony algorithm. Firstly, we proposed the improved optimization path probability formula and the pheromone update formula. Then, we combined the local search algorithm with the ant colony algorithm and put forward the LSACA algorithm. Finally, in the experiment, we studied the parameters setting and the algorithm performance. From the experimental results, we can see that the performance of the LSACA algorithm was better than the ant colony algorithm. And we verify the validity and the feasibility of the LSACA algorithm.

In the future research, we can explore and study the follows aspects. (1) we can research the convergence of ants colony algorithm. Now, the proof of the convergence for ant colony algorithm is not thorough. (2) we can improve the speed of the ant colony algorithm. The speed of ant colony algorithm is always concerned by scholars. (3) we can combine the ant colony algorithm with other algorithm. The hybrid algorithm can improve the performance of the new algorithm. The research on the hybrid algorithm is worth watching.

\section{References}

[1] A. Colorni, M. Dorigo and V. Maniezzo, "Distributed optimization by ant colonies", Proceedings of the 1st European Conference on Artificial Life, (1991), pp. 134-142.

[2] L. M. Gambardella and M. Dorigo, "Ant-Q: A reinforcement learning approach to the traveling salesman problem", Proceedings of the 12st International Conference on Machine Learning, (1995), pp. $252-260$

[3] M. Dorigo, V. Maniezzo and A. Colorni, "Ant system: Optimization by a colony of cooperating agents", IEEE Transactions on System, Man, and Cybernetics-Part B, vol. 26, no. 1, (1996), pp. 29-41.

[4] W. Tfaili and P. Siarry, "A new charged ant colony algorithm for continuous dynamic optimization", Applied Mathematics and Computation, vol. 171, no. 22, (2008), pp. 604-613.

[5] J. Yu, Y. Chen and J. Wu, "Modeling and implementation of classification rule discovery by ant colony optimisation for spatial land-use suitability assessment", Computers, Environment and Urban Systems, vol. 35, (2011), pp. 308-319.

[6] R. F. Tavares Neto and M. Godinho Filho, "An ant colony optimization approach to a permutational flowshop scheduling problem with outsourcing allowed", Computers \& Operations Research, vol. 38, (2011), pp. 1286-1293.

[7] B. Chen, L. Chen and Y. Chen, "Efficient ant colony optimization for image feature selection", Signal Processing, vol. 93, (2013), pp. 1566-1576.

[8] R. Forsati, A. Moayedikia, R. Jensen, M. Shamsfard and M. R. Meybodi, "Enriched ant colony optimization and its application in feature selection [J]", Neurocomputing, vol. 142, (2014), pp. 354371.

[9] G. M. Vásquez and J. Silverman, "Intraspecific aggression and colony fusion in the Argentine ant", Animal Behaviour, (2008), vol. 75, no. 2, pp. 583-593.

[10] C. García-Martínez, O. Cordón and F. Herrera, "A taxonomy and an empirical analysis of multiple objective ant colony optimization algorithms for the bi-criteria TSP", European Journal of perational Research, vol. 180, no. 1, (2007), pp. 116-148.

[11] M. H. Afshar, "Partially constrained ant colony optimization algorithm for the solution of constrained optimization problems: Application to storm water network design", Advances in Water Resources, vol. 30, Issue 4, (2007) April, pp. 954-965.

[12] M. Mavrovouniotis and S. Yang, "Ant colony optimization with immigrants schemes for the dynamic travelling salesman problem with traffic factors", Applied Soft Computing, vol. 13, no. 10, (2013), pp. 4023-4037.

[13] R. M. Rizk-Allah, E. M. Zaki and A. A. El-Sawy, "Hybridizing ant colony optimization with firefly algorithm for unconstrained optimization problems", Applied Mathematics and Computation, vol. 224, no. $1,(\mathbf{2 0 1 3})$, pp. 473-483.

[14] A. Rossi and M. Lanzetta, "Scheduling flow lines with buffers by ant colony digraph", Expert Systems with Applications, vol. 40, no. 9, (2013), pp. 3328-3340.

[15] N. Qingjian, X. Hancheng, Z. Zhibin and W. Qinqin, "Ant colony algorithm and its application review and progress", Computer Application and Software, vol. 8, (2008), pp. 12-16.

[16] H. Han, H. Zhifeng, W. Chunguo and Q. Yong, "The Convergence Speed of Ant Colony Optimization [J]", Chinese Journal of Computers, vol. 8, (2007), pp. 1344-1353. 
[17] X. Yamei, C. Bo, C. Junliang, M. Xiangwu and C. Dong, "Optimizing Services Composition Based on Improved Ant Colony Algorithm”, Chinese Journal of Computers, vol. 2, (2012), pp. 270-281.

[18] D. Haibin, M. Guanjun, W. Daobo and Y. Xiufen, "Improved Ant Colony Algorithm for Solving Continuous Space Optimization Problems", Journal of System Simulation, vol. 19, no. 5, (2007), pp. 974-977.

[19] L. Changan, Y. Xiaohu, L. Chunyang and W. Hua, "Dynamic Path Planning for Mobile Robot Based onImproved Ant Colony Optimization Algorithm", Acta Electronica Sinica, vol. 5, (2011), pp. 12201224.

[20] Y. Zhong, "Research on Several Problems on Basis of Ant Colony Optimization", Southeast University, biomedical engineering, (2005).

[21] Z. Honghao, "Research on the Ant Colony Optimization Algorithm and Its Application", University of Science and Technology of China, pattern recognition and intelligent system, (2006).

[22] L. Bo, "Improvement of Intelligent ant Colony Algorithm and its Application", Yanshan University, Control Theory and Control Engineering, (2010).

[23] B. Jiyun, "Research on Ant Colony Optimization Algorithm and Model of Ant Colony Foraging Behavior" Control Theory and Control Engineering, Control Science and Engineering, (2012). 
International Journal of Signal Processing, Image Processing and Pattern Recognition Vol.9, No.3 (2016) 